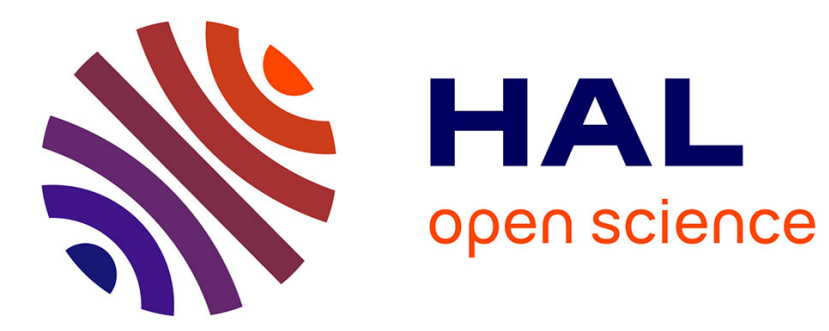

\title{
Beta Current Flow Centrality for Weighted Networks
}

Konstantin Avrachenkov, Vladimir Mazalov, Bulat Tsynguev

\section{To cite this version:}

Konstantin Avrachenkov, Vladimir Mazalov, Bulat Tsynguev. Beta Current Flow Centrality for Weighted Networks. 4th International Conference on Computational Social Networks (CSoNet 2015), Aug 2015, Beijing, China. pp.216-227, 10.1007/978-3-319-21786-4_19 . hal-01258658

\section{HAL Id: hal-01258658 \\ https://hal.inria.fr/hal-01258658}

Submitted on 19 Jan 2016

HAL is a multi-disciplinary open access archive for the deposit and dissemination of scientific research documents, whether they are published or not. The documents may come from teaching and research institutions in France or abroad, or from public or private research centers.
L'archive ouverte pluridisciplinaire HAL, est destinée au dépôt et à la diffusion de documents scientifiques de niveau recherche, publiés ou non, émanant des établissements d'enseignement et de recherche français ou étrangers, des laboratoires publics ou privés. 


\title{
Beta Current Flow Centrality for Weighted Networks
}

\author{
Konstantin E. Avrachenkov ${ }^{1}$, Vladimir V. Mazalov ${ }^{2}$, Bulat T. Tsynguev ${ }^{3}$ \\ 1 INRIA, 2004 Route des Lucioles, Sophia-Antipolis, France \\ k.avrachenkov@sophia.inria.fr \\ 2 Institute of Applied Mathematical Research, Karelian Research Center, \\ Russian Academy of Sciences, 11, Pushkinskaya st., Petrozavodsk, Russia, 185910 \\ vmazalov@krc.karelia.ru \\ 3 Transbaikal State University, 30, Aleksandro-Zavodskaya st., Chita, Russia, 672039 \\ btsynguev@gmail.com
}

\begin{abstract}
Betweenness centrality is one of the basic concepts in the analysis of social networks. Initial definition for the betweenness of a node in a graph is based on the fraction of the number of geodesics (shortest paths) between any two nodes that given node lies on, to the total number of the shortest paths connecting these nodes. This method has quadratic complexity and does not take into account indirect paths. We propose a new concept of betweenness centrality for weighted network, beta current flow centrality, based on Kirchhoff's law for electric circuits. In comparison with the original current flow centrality and alpha current flow centrality, this new measure can be computed for larger networks. The results of numerical experiments for some examples of networks, in particular, for the popular social network VKontakte as well as the comparison with PageRank method are presented.
\end{abstract}

Keywords: beta current flow centrality, betweenness centrality, PageRank, weighted graph, social networks.

\section{Introduction}

The online social networks gave impulse to the development of new graphtheoretical methods for network analysis. Furthermore, social network analysis methods are applied in many other fields such as: economics, physics, biology and information technologies.

One of the basic concepts in the analysis of social networks is betweenness centrality, a measure of centrality that is based on how well a node $i$ is situated in terms of the paths that it lies on [11]:

$$
c_{B}(i)=\frac{1}{n_{B}} \sum_{s, t \in V} \frac{\sigma_{s, t}(i)}{\sigma_{s, t}},
$$

where $\sigma_{s, t}$ is the total number of geodesics (shortest paths) between nodes $s$ and $t, \sigma_{s, t}(i)$ is the number of geodesics between $s$ and $t$ that $i$ lies on. The 
denominator $n_{B}$ captures that the node $i$ could lie on paths between as many as $n_{B}=(n-1)(n-2) / 2$ pairs of other nodes. The complexity of the fastest algorithm to find $c_{B}(i)$ is $O(m n)$ where $m$ is the number of edges and presented in $[8]$.

One of shortcomings of the betweenness centrality is that it takes into accounts only the shortest paths, ignoring the paths that might be one or two steps longer, while the edges on such paths can be important for communication processes in the network. In order to take such paths into account, Brandes and Fleischer [9] and Newman [19] introduced the current flow betweenness centrality (CF-centrality, for short). In $[9,19]$ the graph is regarded as an electrical network with edges being unit resistances. The CF-centrality of an edge is the amount of current that flows through it, averaged over all source-destination pairs, when one unit of current is induced at the source, and the destination (sink) is connected to the ground.

However, the modification proposed in $[9,19]$ comes with a cost. In comparison with the original betweenness centrality, the bottleneck in the computation of CF-centrality is the matrix inversion with complexity $O\left(n^{3}\right)$. To mitigate this high complexity, in [2] the authors suggested a modification of CF-centrality, where in addition to the grounded sink, every node is attached to the ground with some small conductance proportional to the node degree.

The proposal in [2] makes the underlying linear system strongly diagonally dominant and reduces the computational cost of CF-centrality significantly but still needs to apply averaging over all source-destination pairs. In the current work, we go further and suggest to ground all nodes equally, which leads to averaging only over source nodes and reduces further computational cost. We refer to our new method as beta current flow centrality ( $\beta$ CF-centrality, for short).

Additionally, in contrast to the works $[2,9,19]$, we consider weighted networks. Of course, the original betweenness centrality can easily be extended to weighted networks with integer weights. Namely, transform each link of the weight $k$ into $k$ parallel links of weight 1 . We obtain a multigraph. The shortest path between two nodes is determined the same way as in unweighted graph. But the number of geodesics becomes larger because of the multi-links. For instance, if the nodes $i_{1}$ and $i_{2}$ are connected by $k$ links and the nodes $i_{2}$ and $i_{3}$ are connected by $l$ links, then the nodes $i_{1}$ and $i_{3}$ are connected by $k \cdot l$ paths. Applying the formula (1) to the nodes of multigraph we derive the centrality value for weighted graph, but with a very significant increase in computation cost. In the worst case scenario of $k$ links between any two nodes the complexity of the algorithm to find $c_{B}(i)$ is $O\left(m n^{k}\right)$. In contrast, we note that our proposed method has the same computational complexity for weighted and non-weighted graphs.

Finally, we would like to note that, due to its relatively small computational cost, the proposed $\beta \mathrm{CF}$-centrality is very well suited to serve as a characteristic function in the Myerson vector $[1,18]$. The concept of betweenness centrality via the Myerson vector was proposed in [12-14]. Considering the nodes in the network as players and the links as connections between players they formulate 
a communication game. The imputation of the general payoff in this cooperative game can be used for ranking of players and, respectively, for nodes of the graph. In [17] for the communication game with special characteristic function it was proposed a fairly simple imputation procedure based on the generating function and was shown that the resulting imputation agrees with the Myerson value. The advantage of the Myerson value is in taking into account the impact of all coalitions. Using the current flow betweenness centrality as a weight of any subset of the network it is possible to determine a new characteristic function and then rank the nodes as the Myerson value. This approach extends the game-theoretic approach from non-weighted to weighted graphs.

\section{Beta current flow centrality based on Kirchhoff's law}

Consider a weighted graph $G=(V, E, W)$, where $V$ is the set of nodes, $E$ is the set of edges, and $W$ is the matrix of weights, i.e.,

$$
W(G)=\left(\begin{array}{cccc}
0 & w_{1,2} & \ldots & w_{1, n} \\
w_{2,1} & 0 & \ldots & w_{2, n} \\
\vdots & \vdots & \ddots & \vdots \\
w_{n, 1} & w_{n, 2} & \ldots & 0
\end{array}\right)
$$

where $w_{i, j} \geqslant 0$ is weight of the edge connecting the nodes $i$ and $j, n=|V|$ is the number of nodes. Note that $w_{i, j}=0$ if nodes $i$ and $j$ are not adjacent. Here we assume that $G$ is undirected graph, i.e. $w_{i, j}=w_{j, i}$. By random walk interpretation, the method can in fact be extended to directed networks.

Next we introduce the diagonal degree matrix:

$$
D(G)=\left(\begin{array}{cccc}
d_{1} & 0 & \ldots & 0 \\
0 & d_{2} & \ldots & 0 \\
\vdots & \vdots & \ddots & \vdots \\
0 & 0 & \ldots & d_{n}
\end{array}\right)
$$

where $d_{i}=\sum_{j=1}^{n} w_{i, j}$ is the sum of weights of the edges which are adjacent to node $i$ in graph $G$. The Laplacian matrix $L(G)$ for weighted graph $G$ is defined as follows:

$$
L(G)=D(G)-W(G)=\left(\begin{array}{cccc}
d_{1} & -w_{1,2} & \ldots & -w_{1, n} \\
-w_{2,1} & d_{2} & \ldots & -w_{2, n} \\
\vdots & \vdots & \ddots & \vdots \\
-w_{n, 1} & -w_{n, 2} & \ldots & d_{n}
\end{array}\right)
$$

Let the graph $G^{\prime}$ be converted from the graph $G$ by extension with an additional node $n+1$ connected with all nodes of the graph $G$ with the links of constant conductance $\beta$. Thus, we obtain the Laplacian matrix for the modified 
graph $G^{\prime}$ as:

$$
L\left(G^{\prime}\right)=D\left(G^{\prime}\right)-W\left(G^{\prime}\right)=\left(\begin{array}{ccccc}
d_{1}+\beta & -w_{1,2} & \ldots & -w_{1, n} & -\beta \\
-w_{2,1} & d_{2}+\beta & \ldots & -w_{2, n} & -\beta \\
\vdots & \vdots & \ddots & \vdots & \vdots \\
-w_{n, 1} & -w_{n, 2} & \ldots & d_{n}+\beta & -\beta \\
-\beta & -\beta & \ldots & -\beta & \beta n
\end{array}\right) .
$$

Suppose that a unit of current enters into the node $s \in V$ and the node $n+1$ is grounded. Let $\varphi_{i}^{s}$ be the electric potential at node $i$ when an electric charge is located at node $s$. The vector of all potentials $\varphi^{s}\left(G^{\prime}\right)=\left[\varphi_{1}^{s}, \ldots, \varphi_{n}^{s}, \varphi_{n+1}^{s}\right]^{T}$ for the nodes of graph $G^{\prime}$ is determined by the following system of equations (Kirchhoff's current law):

$$
L\left(G^{\prime}\right) \varphi^{s}\left(G^{\prime}\right)=b_{s}^{\prime},
$$

where $b_{s}^{\prime}$ is the vector of $n+1$ components with the values:

$$
b_{s}^{\prime}(i)= \begin{cases}1 & i=s, \\ 0 & \text { otherwise }\end{cases}
$$

The Laplacian matrix (2) is singular. The potential values can be determined up to a constant. Hence, without loss of generality, we can assume that the potential in node $n+1$ is equal to 0 (grounded node). Then, from (3) it follows that

$$
\tilde{\varphi}^{s}\left(G^{\prime}\right)=\tilde{L}\left(G^{\prime}\right)^{-1} b_{s}
$$

where $\tilde{\varphi}^{s}\left(G^{\prime}\right), \tilde{L}\left(G^{\prime}\right)$ and $b_{s}$ are obtained from (3) by deleting the last row and column corresponding to node $n+1$. Notice that in $\varphi^{s}\left(G^{\prime}\right)$ and $b_{s}^{\prime}$ zero elements are deleted. This yields

$$
\tilde{\varphi}^{s}\left(G^{\prime}\right)=[D(G)-W(G)+\beta I]^{-1} b_{s},
$$

where $I$ is a unity matrix of size $n$.

Thus we can consider the vector $\tilde{\varphi}^{s}\left(G^{\prime}\right)$ as the vector of potential values for the nodes of graph $G$, that is,

$$
\tilde{\varphi}^{s}(G)=[L(G)+\beta I]^{-1} b_{s} .
$$

Rewrite (7) in the following form:

$$
\begin{gathered}
\tilde{\varphi}^{s}(G)=[(D(G)+\beta I)-W(G)]^{-1} b_{s}= \\
=\left[I-(D(G)+\beta I)^{-1} D(G) D^{-1}(G) W(G)\right]^{-1}(D(G)+\beta I)^{-1} b_{s} .
\end{gathered}
$$

The matrices $(D(G)+\beta I)^{-1}$ and $(D(G)+\beta I)^{-1} D(G)$ are diagonal with the elements $\frac{1}{d_{i}+\beta}$ and $\frac{d_{i}}{d_{i}+\beta}, i=1, \ldots, n$, denote these matrices as $D_{1}$ and $D_{2}$, respectively. The matrix $D^{-1}(G) W(G)$ is stochastic. Denote it as $P$. Consequently, we have

$$
\tilde{\varphi}^{s}(G)=\left[I-D_{2} P\right]^{-1} D_{1} b_{s}=\sum_{k=0}^{\infty}\left(D_{2} P\right)^{k} D_{1} b_{s}
$$


From (8) it follows that the potential vector can be calculated by the recursion:

$$
\tilde{\varphi}_{k+1}^{s}(G)=D_{2} P \tilde{\varphi}_{k}^{s}(G)+D_{1} b_{s}, \quad \tilde{\varphi}_{0}^{s}(G)=0 .
$$

Note that the convergence is guaranteed since the matrix $D_{2} P$ is substochastic. The rate of convergence can be easily regulated by the value of $\beta$.

The current let-through the link $e=(i, j)$ according to Ohm's law is $x_{e}^{s}=$ $\left|\varphi_{i}^{s}-\varphi_{j}^{s}\right| \cdot w_{i, j}$. Consequently, given that the electric charge is in node $s$, the mean value of the current passing through node $i$ is:

$$
x^{s}(i)=\frac{1}{2}\left(b_{s}(i)+\sum_{e: i \in e} x_{e}^{s}\right),
$$

where

$$
b_{s}(i)= \begin{cases}1 & i=s \\ 0 & \text { otherwise }\end{cases}
$$

Finally, we define beta current flow centrality ( $\beta \mathrm{CF}$-centrality) of node $i$ as follows:

$$
C F_{\beta}(i)=\frac{1}{n} \sum_{s \in V} x^{s}(i) .
$$

We note that the above equation and the law of large numbers can be used to make a simple, light complexity, Monte Carlo type method for quick estimation of $\beta \mathrm{CF}$-centrality. Specifically, we can take a small subset of nodes, $V_{1} \subset V$, chosen independently and uniformly as source nodes in order to approximate $\beta \mathrm{CF}-$ centrality:

$$
C F_{\beta}(i) \approx \frac{1}{\left|V_{1}\right|} \sum_{s \in V_{1}} x^{s}(i)
$$

Let us now investigate the limiting cases of large and small values of $\beta$. First, assume that $\beta$ is large. Then, we can derive the following asymptotics for the potential vector.

$$
\tilde{\varphi}^{s}=[L+\beta I]^{-1} b_{s}=\frac{1}{\beta}\left[I+\frac{1}{\beta} L\right]^{-1} b_{s}=\frac{1}{\beta} b_{s}-\frac{1}{\beta^{2}} L b_{s}+\mathrm{o}\left(\frac{1}{\beta^{2}}\right)
$$

From the above asymptotics, we can conclude that $x^{s}(s)=1 / 2\left(1+d_{s} / \beta\right)+\mathrm{o}(1 / \beta)$ and $x^{s}(i)=\mathrm{o}(1)$, for $i \neq s$, and consequently,

$$
C F_{\beta}(i)=\frac{1}{2 n}+\mathrm{o}(1), \quad \text { as } \quad \beta \rightarrow \infty,
$$

which does not give informative ranking. Now for the other case $\beta \rightarrow 0$, we can derive the following asymptotics

$$
\tilde{\varphi}^{s}=[L+\beta I]^{-1} b_{s}=\left[\frac{1}{\beta} \frac{1}{n} \underline{11}^{T}+L^{\sharp}+\mathrm{O}(\beta)\right] b_{s}=\frac{1}{\beta} \frac{1}{n} \underline{1}+L_{\star, s}^{\sharp}+\mathrm{O}(\beta),
$$


where we have used the Laurent series expansion for inversion of singularly perturbed matrices (see e.g., [4, Chapter 2]) with $\underline{1}$ denoting vector of ones of appropriate dimension, and $L^{\sharp}=\left[L-1 / n \underline{11}^{T}\right]^{-1}-1 / n \underline{11}^{T}$ denoting the group inverse of the Laplacian. Thus, we have

$$
x_{e}^{s}=\left|L_{i, s}^{\sharp}-L_{j, s}^{\sharp}\right| w_{i, j}+\mathrm{o}(1),
$$

and hence a well-defined and non-trivial limit for $\beta \mathrm{CF}$-centrality exists when $\beta \rightarrow 0$.

\section{Illustrative examples}

\subsection{Weighted network of six nodes}

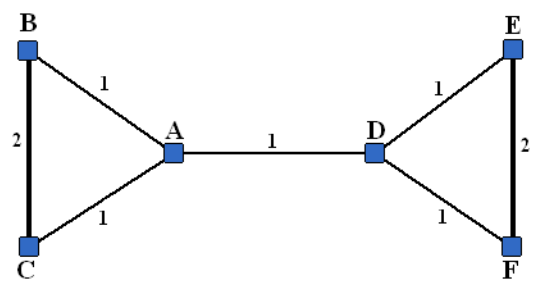

Fig. 1. Weighted network of six nodes.

Let us start with a simple six nodes network example which nicely explains the properties of the beta current flow centrality (see Fig.1). We compute all main measures of centrality for that weighted graph with six nodes. The results of computation are presented in Table 1 . We see that classical betweenness centrality evaluates only the nodes $\mathrm{A}$ and $\mathrm{D}$ and gives 0 to other four nodes, even though they are obviously also important. The PageRank method ranks all nodes with equal values and thus it is indiscriminatory in this particular case. The current flow betweenness centrality and the $\beta \mathrm{CF}$-centrality evaluate all nodes in quite similar manner. In particular, they both give rather high values to nodes $\mathrm{A}$ and $\mathrm{D}$. As we mentioned in the introduction, the comparative advantage of the $\beta$ CF-centrality in its small computational costs.

Table 1. Measures of centrality for weighted graph with six nodes

\begin{tabular}{|c|c|c|c|c|c|c|}
\hline Nodes & $\mathrm{A}$ & $\mathrm{B}$ & $\mathrm{C}$ & $\mathrm{D}$ & $\mathrm{E}$ & $\mathrm{F}$ \\
\hline Original betweenness centrality & 6 & 0 & 0 & 6 & 0 & 0 \\
\hline PageRank centrality $\alpha=0.85$ & $1 / 6$ & $1 / 6$ & $1 / 6$ & $1 / 6$ & $1 / 6$ & $1 / 6$ \\
\hline Current flow betweenness centrality & 1.12 & 0.66 & 0.66 & 1.12 & 0.66 & 0.66 \\
\hline$\beta$ CF-centrality $\beta=1$ & 0.27 & 0.19 & 0.19 & 0.27 & 0.19 & 0.19 \\
\hline
\end{tabular}




\section{$3.2 \quad$ Star graph}

Consider a graph $G$ of $n$ nodes in the form of a star. Let node 1 be the center of the star. The modified Laplacian matrix in this case is given by

$$
\begin{gathered}
\tilde{L}=L+\beta I=D(G)-W(G)+\beta I \\
=\left(\begin{array}{cccc}
n-1+\beta & -1 & \ldots & -1 \\
-1 & 1+\beta & \ldots & 0 \\
\vdots & \vdots & \ddots & \vdots \\
-1 & 0 & \ldots & 1+\beta
\end{array}\right)
\end{gathered}
$$

Its inverse matrix is

$$
\begin{gathered}
\tilde{L}^{-1}=(L+\beta I)^{-1} \\
\beta(1+\beta)(n+\beta) \\
\left.\begin{array}{cccccc}
(1+\beta)^{2} & 1+\beta & 1+\beta & \ldots & 1+\beta \\
1+\beta & 1+\beta(n+\beta) & 1+\beta & \ldots & 1 \\
1+\beta & 1 & 1+\beta(n+\beta) & \ldots & 1 \\
\vdots & \vdots & \vdots & \ddots & \vdots \\
1+\beta & 1 & 1 & \ldots & 1+\beta(n+\beta)
\end{array}\right) .
\end{gathered}
$$

If we take as a source node $s=1$, we find from (9) that

$$
x^{s}(1)=\frac{1}{2}\left(1+\frac{n-1}{n+\beta}\right),
$$

and

$$
x^{s}(i)=\frac{1}{2(n+\beta)}, \quad i=2, \ldots, n .
$$

And for a source node $s \neq 1$, we obtain

$$
\begin{gathered}
x^{s}(1)=\frac{2 n-3+\beta}{2(1+\beta)(n+\beta)}, \\
x^{s}(s)=\frac{1}{2}\left(1+\frac{n-1+\beta}{(1+\beta)(n+\beta)}\right), \\
x^{s}(i)=\frac{1}{2(1+\beta)(n+\beta)}, \quad i \neq 1, s .
\end{gathered}
$$

The latter yields that the $\beta \mathrm{CF}$-centrality for the star graph is

$$
\begin{gathered}
C F_{\beta}(1)=\frac{1}{2 n}\left(1+\frac{n-1}{n+\beta}+(n-1) \frac{2 n-3+\beta}{(1+\beta)(n+\beta)}\right)=\frac{1}{2 n}+\frac{(n-1)(n-1+\beta)}{n(1+\beta)(n+\beta)}, \\
C F_{\beta}(i)=\frac{1}{2 n}\left(\frac{1}{n+\beta}+1+\frac{n-1+\beta}{(1+\beta)(n+\beta)}+(n-2) \frac{1}{(1+\beta)(n+\beta)}\right)
\end{gathered}
$$




$$
=\frac{1}{2 n}+\frac{n-1+\beta}{n(1+\beta)(n+\beta)}, \quad i=2, \ldots, n .
$$

In particular, we can conclude from the above expressions that if $\beta \rightarrow \infty$ all nodes obtain the same value $1 /(2 n)$. And if $\beta \rightarrow 0$ and $n$ is large, the central node obtains a value very close to one and the other nodes have nearly zero value. This is in agreement with the general asymptotics derived in the previous section.

This example also shows that the $\beta$ CF-centrality can be viewed as a flexible characteristic function and thus efficiently used in the calculation of the Myerson vector.

\subsection{The results of computer experiments with online social network VKontakte}

In this subsection we consider the weighted graph extracted from the popular Russian social network VKontakte. The graph corresponds to the online community devoted to game theory. This community consists of 483 participants. As a weight of a link we take the number of common friends between the participants. In fact, the probability that two participants are familiar depends on the number of common friends [14]. This approach is often used in online social networks for link recommendation.

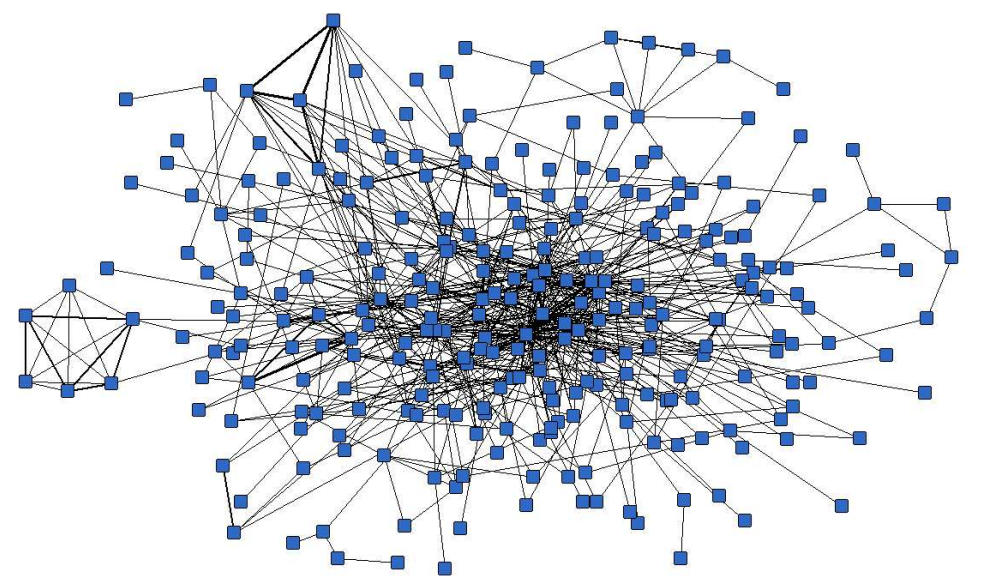

Fig. 2. Principal component of the community Game Theory in the social network VKontakte (number of nodes: 275, number of edges: 805 and mean path's length: $3.36)$.

In Fig. 2 we show the principal component of the community Game Theory, which consists of 275 nodes. It is difficult to see from Fig. 2 which nodes are 


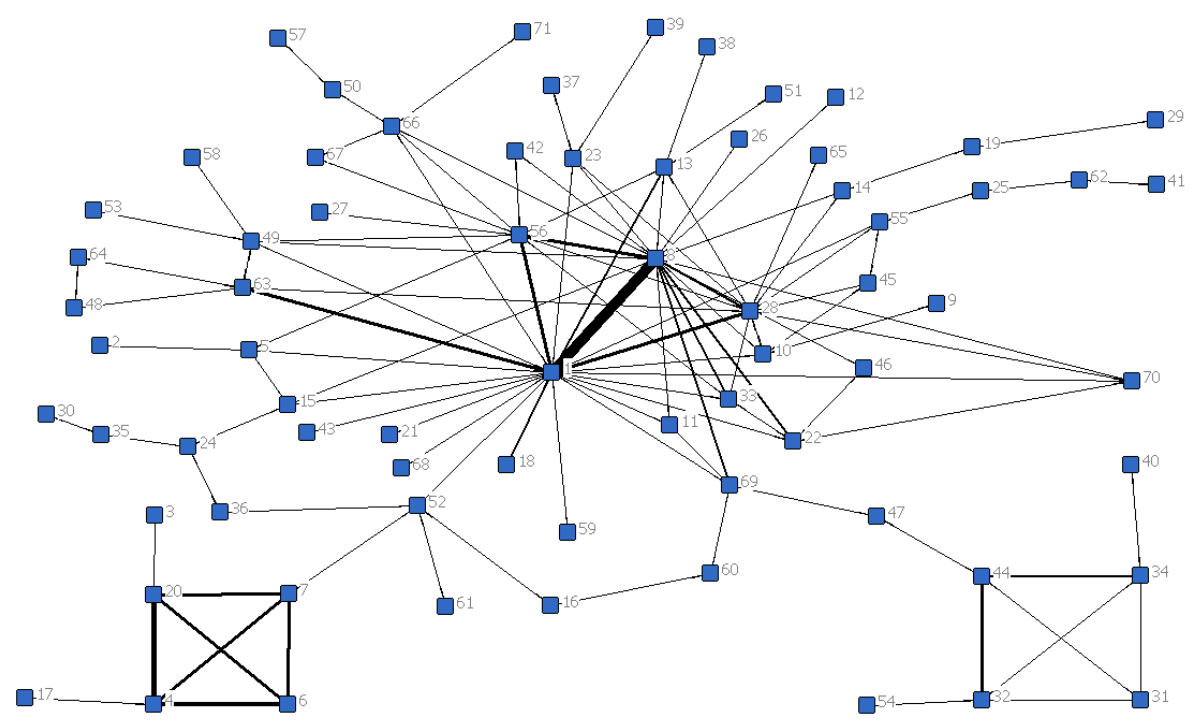

Fig. 3. Principal component of the community Game Theory in the social network VKontakte after deleting the links weighted less than 3 (number of nodes: 71 , number of edges: 116 and mean path's length: 3.75).

more important with respect to the community connection structure. Then, we have converted this graph to another modified graph by deleting the links whose weights are less than three. This new weighted graph is presented in Fig. 3. The thickness of a link depends on the link weight, i.e. on the number of common friends.

The results of computing the $\beta \mathrm{CF}$-centrality for the social network VKontakte are given in Table 2 . Here we take $\beta=0.3$. It is useful to compare these values of $\beta \mathrm{CF}$-centrality with the results corresponding to the PageRank and classical notation of centrality using the shortest paths [20] for the parameter $\alpha=1.5$. We present in the table only the lists of top- 10 nodes for each centrality measure.

From Table 2 we find that all four methods ranked two main nodes 1 and 8 in the same order. We can already see that, as in the six node network example, $\beta \mathrm{CF}$-centrality is more similar to CF-centrality and betweenness centrality than to PageRank.

On Figure 3 we can see that node 52 connects the subgraph $\{3,4,6,7,17,20\}$ with the rest of the graph. Thus, we can expect that node 52 deserves high centrality rank. Similarly, we also expect that node 7 should have high centrality rank. The ranking according to $\beta \mathrm{CF}$-centrality confirms this intuitive expectation, as they take positions 3 and 7, respectively (See Table 2). We also note that nodes 4, 20,6, 17 and 3 took positions 22, 24,36, 68 and 69, respectively. However, PageRank gives to nodes 52 and 7 only positions 22 and 12, respec- 
tively. Furthermore, under PageRank ranking nodes 4, 20 and 6 took positions 6,8 and 10, respectively. Namely, PageRank ranks nodes 4, 20 and 6 higher than node 52 . This does not correspond at all to our intuition.

Finally, in Table 3 we present the results of the Monte Carlo approach (see equation (11)) with sampling only $10 \%$ of the nodes as sources. Nodes 1 and 8 as before lead the ranking and there are 6 correct elements in the top-10 basket of nodes [3]. Monte Carlo approach also determines correctly the ranks of the key nodes 52 and 7.

\section{Conclusion}

Betweenness centrality measure is an important tool in the analysis of social networks. The structure of a network is represented by a graph. The original betweenness centrality measure is based on the assumption that the information is transmitted along geodesics (shortest paths) between any two nodes. There is a criticism of this approach that it does not take into account information spread along non-shortest paths. The current flow betweenness centrality based on electric circuit interpretation tries to mitigate this shortcoming. However, this comes with the increase of computational cost. We introduce here the $\beta \mathrm{CF}$ centrality method which depends on the parameter $\beta$. This method is versatile, has lower computational complexity and can be easily used as characteristic function in the Myerson vector.

Table 2. Measures of centrality for top nodes of social network VKontakte.

\begin{tabular}{|c|c|c|c|c|c|c|c|}
\hline Nodes & $\begin{array}{c}\beta \mathrm{CF} \text {-centrality } \\
(\beta=0.3)\end{array}$ & Nodes & $\begin{array}{l}\text { PageRank } \\
\text { centrality } \\
(\alpha=0.85)\end{array}$ & Nodes & $\begin{array}{c}\text { Weighted } \\
\text { betweenness } \\
\text { centrality "tnet" } \\
(\alpha=1.5)\end{array}$ & Nodes & CF-centrality \\
\hline 1 & 0.4168 & 1 & 0.1359 & 1 & 1846 & 1 & 0.6406 \\
\hline 8 & 0.3143 & 8 & 0.1189 & 8 & 1398 & 8 & 0.4919 \\
\hline 52 & 0.1463 & 56 & 0.0432 & 52 & 500 & 69 & 0.2946 \\
\hline 69 & 0.1454 & 28 & 0.0366 & 69 & 494 & 52 & 0.2748 \\
\hline 28 & 0.1299 & 44 & 0.0277 & 47 & 384 & 28 & 0.2095 \\
\hline 56 & 0.1273 & 4 & 0.0267 & 44 & 331 & 56 & 0.1942 \\
\hline 7 & 0.1002 & 32 & 0.0252 & 63 & 331 & 47 & 0.1880 \\
\hline 15 & 0.0931 & 20 & 0.0244 & 7 & 325 & 44 & 0.1649 \\
\hline 66 & 0.0922 & 63 & 0.0228 & 55 & 265 & 15 & 0.1645 \\
\hline 63 & 0.0896 & 6 & 0.0212 & 15 & 228 & 7 & 0.1642 \\
\hline
\end{tabular}


Table 3. The results of the Monte Carlo approach with sampling only $10 \%$ of the nodes as sources

\begin{tabular}{|c|c||c|c|}
\hline Nodes & $\begin{array}{c}\beta \text { CF-centrality } \\
(\beta=0.3)\end{array}$ & Nodes & $\begin{array}{c}\text { Monte Carlo } \\
\text { approach } \\
10 \% \text { of the nodes }\end{array}$ \\
\hline 1 & 0.4168 & 1 & 0.5043 \\
5 & 0.3143 & 8 & 0.4134 \\
52 & 0.1463 & 52 & 0.2468 \\
28 & 0.1454 & 23 & 0.2307 \\
56 & 0.1299 & 28 & 0.2255 \\
7 & 0.1273 & 20 & 0.2003 \\
15 & 0.1002 & 7 & 0.1982 \\
66 & 0.0931 & 24 & 0.1871 \\
63 & 0.0922 & 63 & 0.1789 \\
47 & 0.0896 & 10 & 0.1786 \\
24 & 0.0889 & 15 & 0.1763 \\
44 & 0.0880 & 55 & 0.1756 \\
55 & 0.0842 & 36 & 0.1613 \\
49 & 0.0801 & 69 & 0.1565 \\
23 & 0.0725 & 12 & 0.1457 \\
13 & 0.0702 & 39 & 0.1438 \\
10 & 0.0610 & 56 & 0.1397 \\
14 & 0.0598 & 3 & 0.1360 \\
25 & 0.0564 & 4 & 0.1234 \\
\hline
\end{tabular}

\section{Acknowledgements}

This research is supported by Russian Humanitarian Science Foundation (project 15-02-00352), the Division of Mathematical Sciences of Russian Academy of Sciences, EU Project Congas FP7-ICT-2011-8-317672 and Campus France. 


\section{References}

1. Aumann, R., Myerson, R.: Endogenous formation of links between players and coalitions: an application of the Shapley value. In: The Shapley value, Cambridge University Press, pp.175-191 (1988)

2. Avrachenkov, K., Litvak, N., Medyanikov, V., Sokol, M.: Alpha current flow betweenness centrality. In Proceedings of WAW 2013, LNCS v.8305, pp.106-117 (2013)

3. Avrachenkov, K., Litvak, N., Nemirovsky, D., Smirnova, E., Sokol, M.: Quick detection of top-k personalized pagerank lists. In Proceedings of WAW 2011, LNCS v.6732, pp.50-61 (2011)

4. Avrachenkov, K.E., Filar J.A., Howlett, P.G.: Analytic Perturbation Theory and its Applications, SIAM (2013).

5. Borgatti, S.P., Everett, M.G., Freeman, L.C.: Ucinet for Windows: Software for Social Network Analysis. Harvard (2002)

6. Borm, P., Owen, G., Tijs, S.: On the position value for communication situations. SIAM J. on Disc. Math., v.5(3), pp.305-320 (1992)

7. Borm, P., van den Nouweland, A., Tijs, S.: Cooperation and communication restrictions: a survey. In: Imperfections and Behavior in Economic Organizations. Kluwer (1994)

8. Brandes, U.: A faster algorithm for betweenness centrality. Journal of Mathematical Sociology, v.25, pp.163-177 (2001)

9. Brandes, U., Fleischer, D.: Centrality measures based on current flow. In Proceedings of the 22nd annual conference on Theoretical Aspects of Computer Science, pp.533-544 (2005)

10. Calvo, E., Lasaga, J., van den Nouweland, A.: Values of games with probabilistic graphs. Math. Social Sci., v.37, pp.79-95 (1999)

11. Freeman, L.C.: A set of measures of centrality based on betweenness. Sociometry, v.40, pp.35-41 (1977)

12. Jackson, M.O.: Allocation rules for network games. Games and Econ. Behav., v.51(1), pp.128-154 (2005)

13. Jackson, M.O., Wolinsky, J.: A strategic model of social and economic networks. J. Econ. Theory, v.71(1), pp.44-74 (1996)

14. Jackson, M.O.: Social and economic networks. Princeton University Press (2008)

15. Jamison, R.E.: Alternating Whitney sums and matchings in trees. Part 1. Disc. Math., v.67, pp.177-189 (1987)

16. Mazalov, V.: Mathematical Game Theory and Applications. Wiley (2014)

17. Mazalov, V.V., Trukhina, L.I.: Generating functions and the Myerson vector in communication networks. Disc. Math. and Appl. v.24(5), pp.295-303 (2014)

18. Myerson, R.B.: Graphs and cooperation in games. Math. Oper. Res., v.2, pp.225$229(1977)$

19. Newman, M.E.J.: A measure of betweenness centrality based on random walks. Social networks, v.27, pp.39-54 (2005)

20. Opsahl, T., Agneessens, F., Skvoretz, J.: Node centrality in weighted networks: generalizing degree and shortest paths. Social Networks, v.32, pp.245-251 (2010)

21. Slikker, M.: Link monotonic allocation schemes. Int. Game Theory Review, vol. 7(4), pp.473-489 (2005)

22. Slikker, M., Gilles, R.P., Norde, H., Tijs, S.: Directed networks, allocation properties and hierarchy formation. Math. Social Sci., v.49(1), pp.55-80 (2005)

23. Talman, D., Yamamoto, Y.: Average tree solutions and subcore for acyclic graph games. J. Oper. Res. Soc. Japan, v.51(3), pp.187-201 (2008) 